\title{
LISA and its precursor LISA Pathfinder
}

\author{
A. F. García Marín ${ }^{* 1}$, G. Heinzel ${ }^{1}$ and K. Danzmann ${ }^{1}$ on behalf of the LISA \\ community \\ ${ }^{1}$ Max-Planck-Institut für Gravitationsphysik (Albert-Einstein-Institut) and Universität Hannover, \\ Callinstr. 38, D-30167 Hannover, Germany \\ E-mail: antonio.garcia@aei.mpg.de, gerhard.heinzel@aei.mpg.de, \\ Karsten.Danzmann@aei.mpg.de,
}

The Laser Interferometer Space Antenna (LISA) is a joint ESA-NASA mission designed to observe gravitational waves in the frequency range between $10^{-4}$ to $1 \mathrm{~Hz}$, where ground-based detectors are limited by terrestrial noise. Sources in this frequency range include supermassive black holes and galactic binary stars. LISA consists of three identical spacecraft separated by 5 million kilometers carrying a total of six free flying proof masses in heliocentric drag-free orbit. The fluctuations in separation between two test masses located in different satellites will be measured by laser interferometry with picometer precision. LISA Pathfinder is a technology demonstration mission for LISA consisting of only two test masses in one single satellite. It will be launched in 2009, five years before LISA. We provide here an overview of the development of LISA and LISA Pathfinder with particular emphasis on the interferometry.

PACS: $07.60 . \mathrm{LY}, 95.55 . \mathrm{Ym}, 95.75 . \mathrm{Kk}$

International Europhysics Conference on High Energy Physics

July 21st - 27th 2005

Lisboa, Portugal

* Speaker. 


\section{Introduction}

LISA [1] will be the first gravitational wave observatory in the low frequency range, where astrophysical events of high energy are expected. LISA is fi rmly established in the future programs of both ESA and NASA, and its technology demonstrator, LISA pathfi nder, has already entered its implementation phase.

\section{LISA}

The LISA constellation is shown in Figure 1. The three satellites form an equilateral triangle that orbits the sun following the earth in a distance of 50 Million kilometer. This orbit provides a high stability environment for the free fbating test masses, so that two of them placed in different satellites can be used as end mirrors of a high precision interferometer to detect gravitational waves.

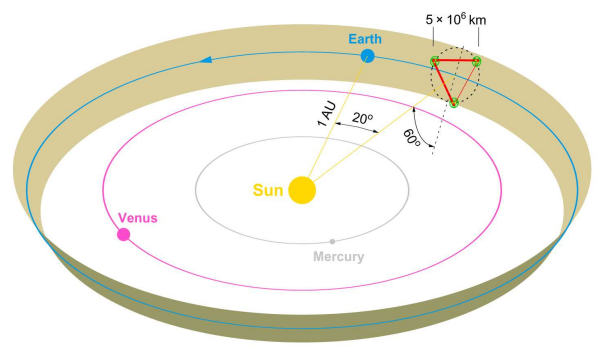

Figure 1: The three LISA satellites orbit the sun 20 degrees behind the earth in a equilateral triangle formation.

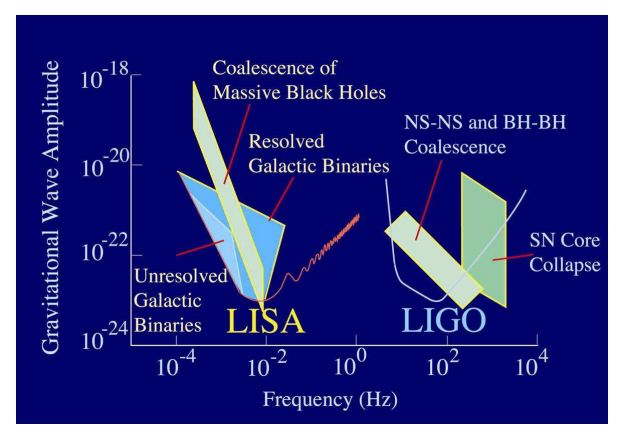

Figure 2: LISA's dimensionless gravitational wave strain sensitivity for a signal to noise ratio (SNR) of 5 and one year integration time.

LISA will achieve a sensitivity to gravitational wave strain $\frac{\delta l}{L}$ in the millihertz range (Figure 2) comparable to that of the ground base detectors at higher frequencies. Several technical challenges have to be met to achieve this sensitivity:

Drag free control system: it must keep the residual acceleration of each test mass below $3 \times 10^{-15} \mathrm{~ms}^{-2} / \sqrt{\mathrm{Hz}}$ at $3 \mathrm{mHz}$.

Interferometry: its sensitivity has to be better than $40 \times 10^{-12} \mathrm{~m} / \sqrt{\mathrm{Hz}}$ at $3 \mathrm{mHz}$.

Micronewton Thrusters: they must provide with very low noise the continuous forces of micronewton magnitude that are necessary for the drag-free operation.

A in-flght test for LISA was designed to test these technologies.

\section{LISA Pathfinder}

The LISA pathfi nder mission (LPF) [2] consists of only one satellite (see Figure 3) carrying the European LISA Technology Package (LTP) as payload, and in addition a second set of thrusters with its drag free control system supplied by the US. 


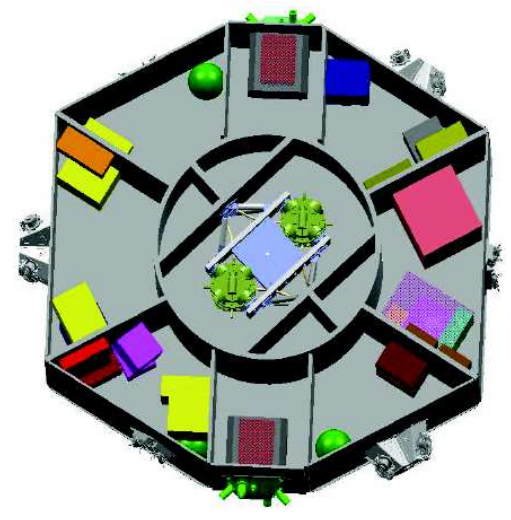

Figure 3: LISA Pathfinder satellite. The LTP is shown in the center of the satellite.

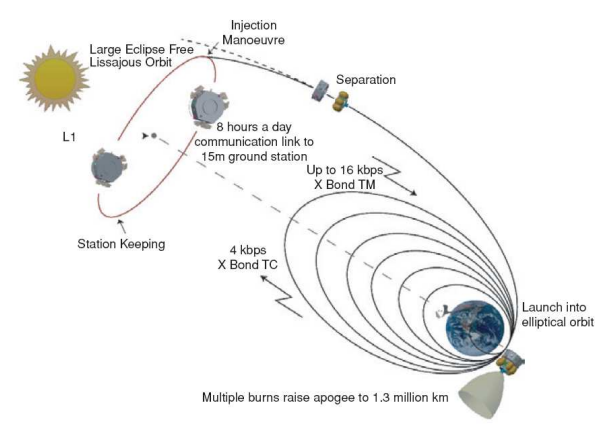

Figure 4: LISA Pathfinder will orbit the first Lagrange point.

The aim of the mission is to verify in space the LISA core technologies mentioned in section 2 , mainly the drag-free control system. For its implementation, the satellite carries two free fbating LISA-like test masses whose relative motion along a common sensitive axis is measured by means of a laser interferometer (see Figure 5). The position and orientation of each test mass with respect to the satellite is additionally measured by a set of electrodes that surround it. The eighteen degrees of freedom of the satellite and the two test masses are separated into "drag-free" coordinates, which are actuated by the micronewton thrusters, and the "suspension coordinates" for which the capacitive electrodes are used as actuators [2].

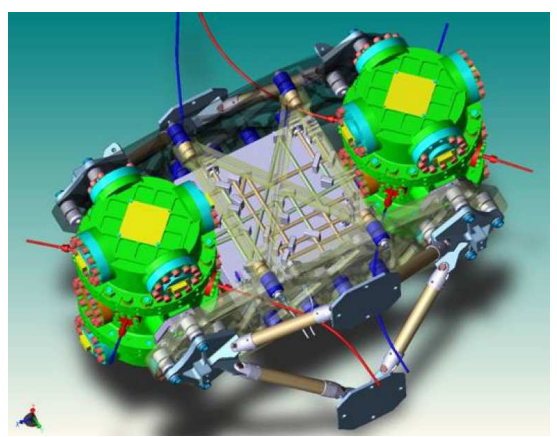

Figure 5: LPF core assembly. Two vertical Zerodur plates hold the optical bench and the two vacuum tanks containing the test masses. Laser light from the optical bench is reflected on the test masses allowing the interferometrical determination of their position fluctuations.

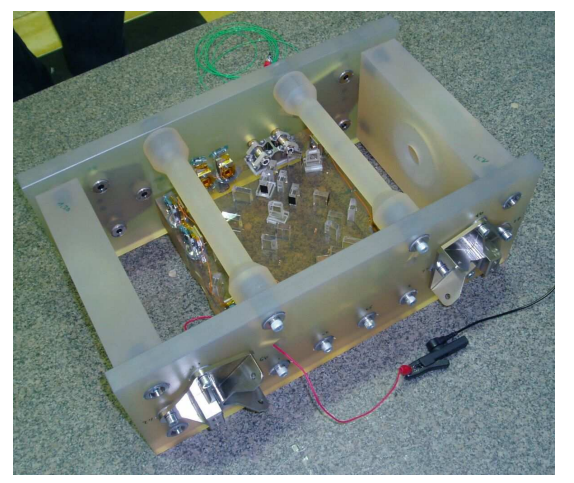

Figure 6: Engineering model of the optical bench. The test masses have been substituted by gold-coated mirrors for ground testing.

\subsection{LISA pathfinder interferometry}

A set of four non-polarizing heterodyne Mach-Zehnder interferometers will be used onboard LISA Pathfi nder to detect position fluctuations of the test masses. Figure 7 illustrates their principle of function $[3,4,5]$ : the laser is split into two different beams and each of them is frequency shifted 
by an acousto-optical modulator (AOM). The two RF frequencies that drive the AOMs differ by a constant amount of about $1 \mathrm{kHz}$, the heterodyne frequency $f_{\text {het }}$. These two frequency shifted beams are then coupled into optical fi bers and brought onto an optical bench which is made out of Zerodur for high thermal stability [6]. On the bench, the incoming beams are again split and fi nally brought to interference in at least two different beamsplitters (Reference and one or more Measurement).

On the actual LTP optical bench (Figure 8) the arms of the reference interferometer recombine directly in a beamsplitter while one of the beams of the measurement interferometer is reflected from both test mass before recombining. In addition, there are two more interferometers that are used to monitor the second test mass independently and to stabilize the laser frequency, respectively $[3,4,7]$.

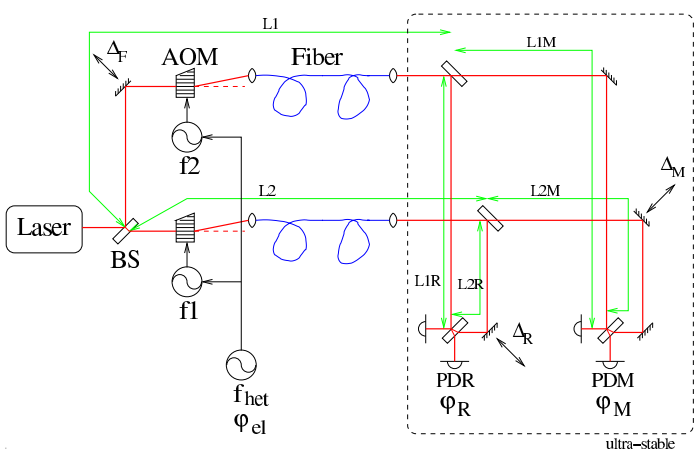

Figure 7: Simplified schema of the interferometry concept for the LISA technology package. Only one measurement interferometer shown.

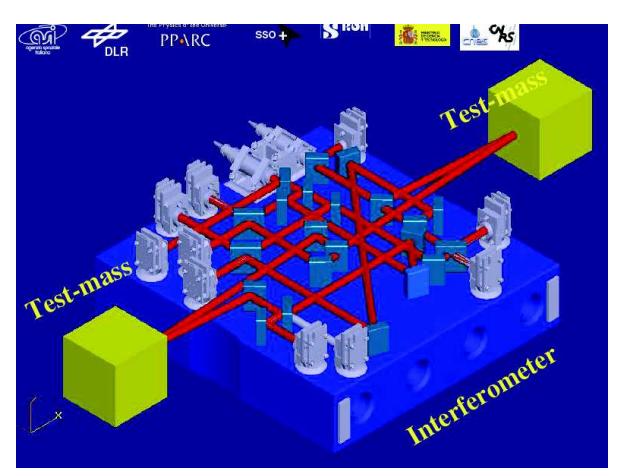

Figure 8: Actual layout of the ultra-stable LTP optical bench.

The heterodyne signal detected by a photodiode at the output port of each interferometer is given by:

$$
\mathrm{I}(t)=\mathrm{A}\left(1-\mathrm{c} \cos \left(2 \pi f_{\text {het }}+\varphi(t)\right)\right)
$$

where $\mathrm{I}$ is the measured intensity, A the average photocurrent of the heterodyne signal and $\mathrm{c}$ the interferometric contrast. The phase $\varphi$ of the Reference and Measurement signals (Figure 7) can be written as:

$$
\begin{gathered}
\varphi_{\mathrm{R}}=\frac{2 \pi}{\lambda}\left(\mathrm{L}_{1}-\mathrm{L}_{2}+\mathrm{L}_{1 \mathrm{R}}-\mathrm{L}_{2 \mathrm{R}}\right)=\Delta_{\mathrm{F}}+\Delta_{\mathrm{R}} \\
\varphi_{\mathrm{M}}=\frac{2 \pi}{\lambda}\left(\mathrm{L}_{1}-\mathrm{L}_{2}+\mathrm{L}_{1 \mathrm{M}}-\mathrm{L}_{2 \mathrm{M}}\right)=\Delta_{\mathrm{F}}+\Delta_{\mathrm{M}}
\end{gathered}
$$

where $\Delta \mathrm{F}$ represents the (large) common-mode pathlength variations present in both interferometers, and $\Delta_{R}$ and $\Delta_{M}$ the pathlength variations on the stable optical bench of the Reference and Measurement interferometer, respectively. The main interferometrical measurement of LTP consists of a measurement of the photocurrent phase in a multichannel electronic phasemeter, and the subsequent substraction between $\varphi_{\mathrm{R}}$ and $\varphi_{\mathrm{M}}$, as this cancels the common fluctuations $\Delta_{\mathrm{F}}$ and only the position fluctuations of the test mass remain $[8,9]$.

Measurements on the engineering model of the optical bench (see Figure 6) have produced the noise measurement shown in Figure 9, where the noise specification allocated for the determination of the phase difference was met. Furthermore, the use of quadrant photodiodes (QPD) 


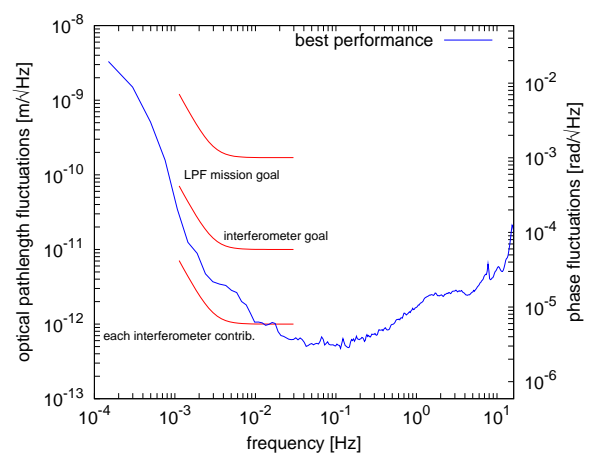

Figure 9: Sensitivity of the LTP interferometer and phasemeter for longitudinal test mass displacement.

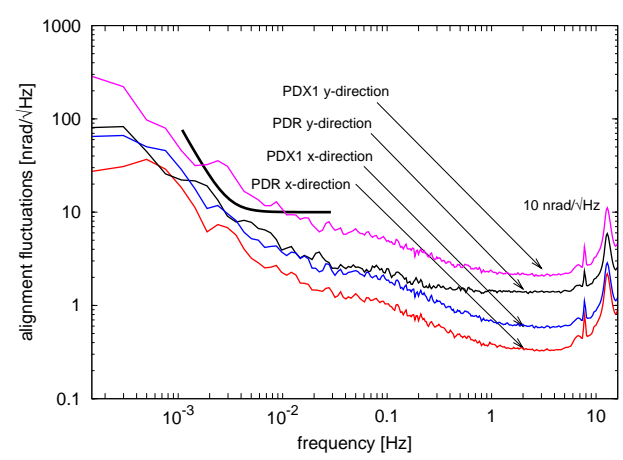

Figure 10: Sensitivity of the LTP interferometer and phasemeter for test mass tilt and rotation.

allows the determination of tilt and rotations of the test masses. Figure 10 shows the noise curves corresponding to these angle determination, which also fulfi 11 the requirements.

\section{References}

[1] LISA Study Team 1998 Max-Plank-Institut für Quantenoptik MPQ233

[2] Anza S et al 2005 Class. Quantum Grav. 22 125-138

[3] Heinzel G et al 2003 Class. Quantum Grav. 20 153-161

[4] Heinzel G et al 2004 Class. Quantum Grav. 21 581-587

[5] Heinzel G et al 2005 Class. Quantum Grav. 22 149-154

[6] Robertson D et al 2005 Class. Quantum Grav. 22 155-163

[7] García Marín A F et al 2006 to appear in Class. Quantum Grav.

[8] Heinzel G et al 2006 to appear in J. Phys.: Conf. Series

[9] Wand V et al 2006 to appear in Class. Quantum Grav. 